\title{
Characterization of MOS structure using low-k dielectric methylsilsesquioxane with evaporated and sputtered aluminium gate
}

\author{
K.C. Aw ${ }^{1)}$, K. Ibrahim \\ School of Applied Physics, University Science of Malaysia, Penang 11800, Malaysia \\ Phone: 6-(04)-636-6157; fax: 6-(04)-636-6500') \\ email:kcaw@altera.com ${ }^{1)}$,kamarul@usm.my
}

\begin{abstract}
The organic methylsilsesquioxane (MSQ) demonstrates low dielectric constant value (2.6) and is promising interlayer dielectric material to reduce the capacitive coupling between metal layers in semiconductor integrated circuits. However, MSQ has lower film density and therefore is more porous than the traditional $\mathrm{SiO}_{2}$ film and could pose reliability issues. This paper is aimed to characterize the MOS capacitor (MOSC) structure with evaporated and sputtered aluminium method deposited on top of spin-on MSQ. Electrical characterization using $\mathrm{C}-\mathrm{V}$ and I-t measurements during bias temperature stress (BTS) were used to understand the effect of evaporated and sputtered Al on MSQ. The results show that MOSC with evaporated aluminium has lower breakdown voltage and has poor reliability as compared to structures with sputtered aluminium. The high temperature required for evaporation compared to sputtering process caused these, which cause defects at the aluminium/MSQ interface. Sputtered aluminium gate structures demonstrate $\mathrm{Al}^{+}$injection under high positive voltage stress due to ionization at the Al/MSQ interface.
\end{abstract}

Keywords: methylsilsesquioxane, low dielectric constant, aluminium, evaporation, sputtering

Paper received 07.06.02; accepted for publication 10.12.02.

\section{Introduction}

Device dimension is being scaled down and the dielectric constant (k) of interlayer dielectric (ILD) of multilevel interconnections in VLSI devices needs to be reduced to improve the speed performance. However, low-k dielectric materials such as MSQ using spin-on process have several problems, such as diffusion [1], which affect the reliability of VLSI devices. This paper concentrates on the characterization of MSQ400F produced by Filmtronics. Filmtronics 400F Methylsilsesquioxane (MSQ) spin-on glass is based on a unique chemistry that yields a polymer with $\mathrm{Si}-\mathrm{CH}_{3}$ and $\mathrm{Si}-\mathrm{O}$ bonds and has a low dielectric constant value (2.6).

Deposition process with aluminium metal using filament evaporation process and sputtering process were employed in this research. Fabricated MOS capacitors (MOSC) with aluminium gate was used in this study. $\mathrm{C}-\mathrm{V}$ measurements and leakage current during bias temperature stress (BTS) were performed to understand the mechanism in the MOSC structure with evaporated and sputtered aluminium gate.

\section{Experimental}

Simple MOSC structure was used to study the electrical stability of MSQ used as dielectric material. The starting material is a $p$-type, $<100>$-silicon substrate. MSQ was deposited using spin-on technique based on a spinner. The thickness of the MSQ can be determined through the speed of the spinner and in this paper is $4000 \AA$. The deposited MSQ was then soft baked to drive out moisture by baking in three stages at $100^{\circ} \mathrm{C}$ for 2 minutes, $180^{\circ} \mathrm{C}$ for 2 minutes and then $200{ }^{\circ} \mathrm{C}$ for 1 minute. The MSQ was then cured in $\mathrm{N}_{2}$ ambient at $400{ }^{\circ} \mathrm{C}$ for 30 minutes [2]. Two types of MOSC structures were fabricated as shown in Fig. 1, i.e. MOSC-E with evaporated Al gate and MOSC-S with sputtered Al gate. Post deposition annealing at $300^{\circ} \mathrm{C}$ for 30 minutes in $N_{2}$ ambient was then performed.

The MOSC structures were subjected to bias-temperature-stressing (BTS) [3] with $+10 \mathrm{~V}$ at $85^{\circ} \mathrm{C}$ to study the stability of the structure over time. This was performed using the HP Semiconductor Parameter Analyser. The high-frequency $(100 \mathrm{KHz}) \mathrm{CV}$ plots were also taken for 
K.C. Aw et al.: Characterisation of MOS structure using low - k dielectric methylsilsesquioxane...
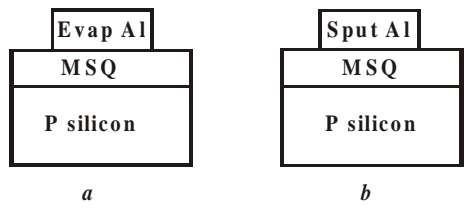

$b$

Fig. 1. Cross section of MOSC structure used in this study (a) MOSC with evaporated aluminium and $(b)$ MOSC with sputtered aluminium

each MOSC structure using Kiethley CV/IV measurement unit. CV plots after constant high voltage positive or negative stresses were also taken. All measurements were carried out in a light tight Faraday box using Micromanipulator prober with a thermal chuck.

\section{Results and discussion}

The leakage current at room temperature $\left(25^{\circ} \mathrm{C}\right)$ is on average approximately $2.5 \cdot 10^{-10} \mathrm{~A} / \mathrm{cm}^{2}$. However, this current will increase with temperature. Fig. 2 shows the leakage current of MOSC-E and MOSC-S with respect to temperature. The leakage current of MOSC-E increases more rapidly with temperature than MOSC-S. This indicates that the A1/MSQ interface of MOSC-E is inferior compare to MOSC-S as Al evaporation occurs at much higher temperature than sputtering process.

Fig. 3 shows the leakage current through the MSQ for MOSC-E and MOSC-S subjected to BTS. Fig. 3 also shows that the leakage current of MOSC-S decreases with stress time whereas the leakage current for MOSC-E remains constant. This reduction of leakage current in MOSC-S suggested that there is a build up of positive charge in the MSQ and reduce the rate of positive charge injection. $\mathrm{C}-\mathrm{V}$ measurements could not be performed on MOSC-E since the breakdown voltage is too low which is in the range of $0.3 \mathrm{MV} / \mathrm{cm}$ [4]. However, CV measurement on MOSC-S samples in Fig. 4 shows that there is a negative shift in the flat band after $+20 \mathrm{~V}$ stress at the gate at room temperature, which confirms positive charge trapped in the MSQ after positive voltage stress. Fig. 5, shows negligible positive shift in the flat-band voltage

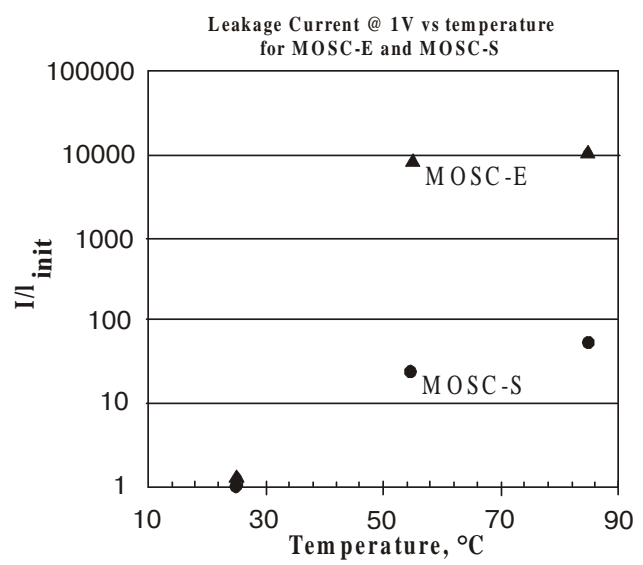

Fig. 2. Leakage current with respect to temperature

SQO, 5(3), 2002

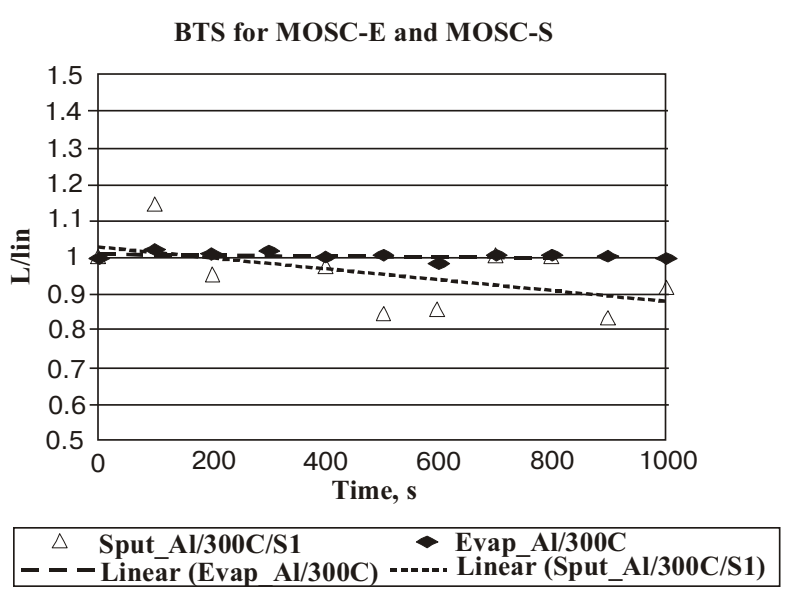

Fig. 3. I versus $t$ for MOSC-E and MOSC-S during BTS

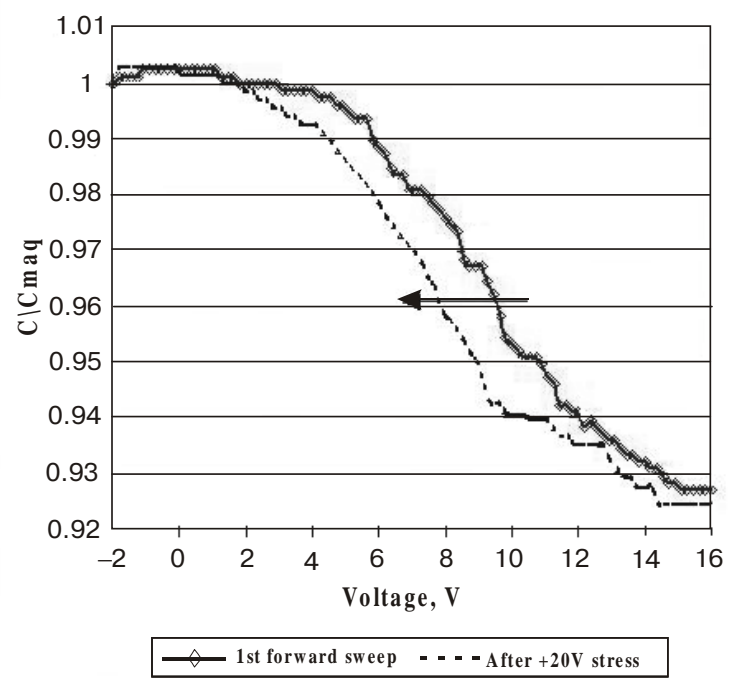

Fig. 4. CV-plot of MOSC-E after $+20 \mathrm{~V}$ stress at the gate

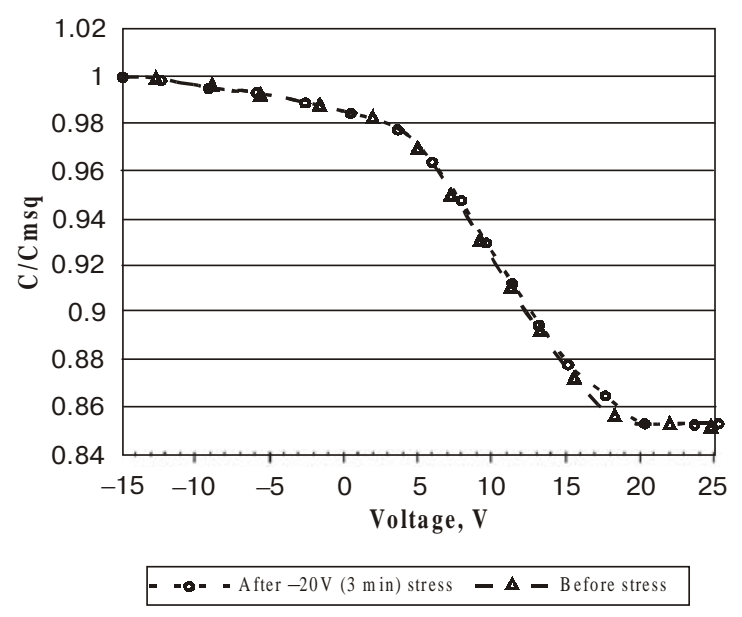

Fig. 5. CV-plot of MOSC-E after $-20 \mathrm{~V}$ stress at the gate

after $-20 \mathrm{~V}$ stress at the gate which implies that the applied voltage is insufficient to induce polarization charge at the MSQ/Si interface. 


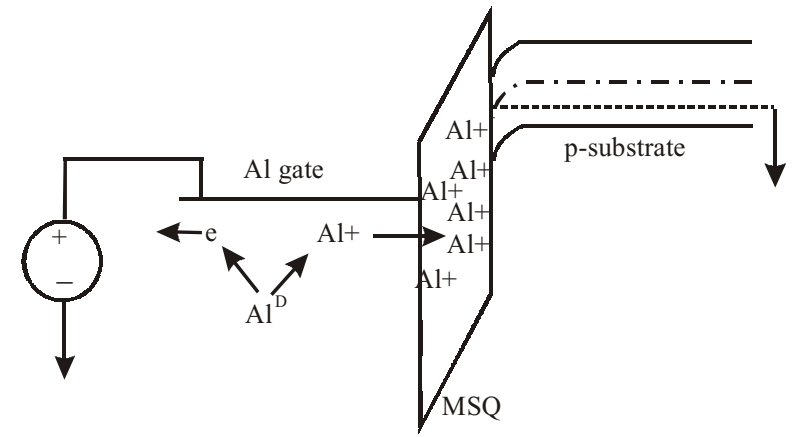

Fig. 6. Proposed model depicting the kinetics of $\mathrm{Al}+$ penetration under positive gate voltage

\section{Summary and conclusions}

This research proves that $\mathrm{Al}$ deposition using evaporation technique is not suitable for MSQ as it demonstrates low breakdown voltage and high leakage current at high temperatures. Al sputtering technique is a more suitable deposition process as it provides higher breakdown voltage and lower leakage current. The main difference in the evaporation and sputtering deposition method that is critical to the MSQ is the deposition temperature. Evaporation of $\mathrm{Al}$ requires about $1000{ }^{\circ} \mathrm{C}$, which is about 4 times higher than that of sputtering process. This high temperature is beyond the decomposition temperature of MSQ, which is lower than $500{ }^{\circ} \mathrm{C}$. This explains the reason why MOSC structure with evaporated Al (MOSCE) is not suitable for integration with MSQ. These research observations also suggest that MOSC with sputtered $\mathrm{Al}(\mathrm{MOSC}-\mathrm{S})$ has $\mathrm{Al}^{+}$that contribute to the positive charge injection. The $\mathrm{Al}^{+}$could be generated from the ionization [5] of neutral Al atoms at the gate/MSQ interface, which is then injected towards the MSQ while leaving behind electrons, which are collected by the voltage source. The proposed physical model described in Fig. 6 governs the drift kinetics of $\mathrm{Al}^{+}$.

\section{Acknowledgement}

The authors would like to thank Dr. Mat Johar and Miss Ee Bee Choo of University Science Malaysia for their help with the CV-meter measurement and clean-room support, respectively. The authors would also like to thank Altera Corporation, Penang for the use of microprobing, HP Semiconductor Parameter Analyser, and Chemical Lab facility.

\section{References}

1. KC Aw et al, The Study of MSQ SOG as Low - K Dielectric Material used as ILD in Semiconductor Devices, MASS XVIII Sept 2001, pp. 18.

2. Filmtronics Application Notes on Spin-on Glasses (MSQ400F), Revision No. 5, June 1998.

3. Bing-Yue Tsui et al, Electrical Instability of Low-Dielectric Constant Diffusion Barrier Film (a-SiC:H) for Copper Interconnect, IEEE Trans on Electron Devices, Vol.48,No.10, Oct 2001, pp. 2376.

4. K.C. Aw et al, Comparative Study of Evaporated Aluminium and Copper on MSQ low-K Dielectric using Spin-on Method, NSM2001, Nov 2001, pp 108.

5. Alvin L.S. Loke et al, Kinetics of Copper Drift in Low-K Polymer Interlevel Dielectrics, IEEE Trans on Electron Devices, Vol.46, No.11, Nov 1999, pp. 2182-2183. 